\title{
Comment on the Scattering-Matrix Method for Determining Defect Modes*
}

\author{
R. E. Allen \\ Department of Physics, University of Texas, Austin, Texas 78712 \\ (Received 25 June 1970)
}

\begin{abstract}
It is pointed out that the formulation of the scattering-matrix method given by Achar and Barsch, which was proposed and used for calculating localized modes at surfaces and other planar defects, is based on an incorrect assumption.
\end{abstract}

A formulation of the scattering-matrix method for calculating localized modes at any type of planar defect has been given by Achar and Barsch ${ }^{1}$ and applied to the problem of determining the surface modes of a semi-infinite $\mathrm{NaCl}$ crystal. ${ }^{2}$ Here we will consider only the application of this formulation when the defect is a surface. Similar criticisms hold for other types of defects.

Achar and Barsch assume that the displacements associated with a surface mode have the form

$$
u_{i}(\overrightarrow{\mathrm{m}} \mu)=\beta_{i}(\mu) e^{i k_{3}\left[\mathrm{x}_{3}\left(m_{3}\right)+\mathrm{x}_{3}(\mu)\right]} e^{i \overrightarrow{\mathrm{k}}_{\rho} \circ \overrightarrow{\mathrm{R}}_{\rho}(\vec{m})},
$$

outside the region perturbed by the surface, where the notation is defined in Refs. 1 and 2, and $k_{3}$ is purely imaginary. This assumption is incorrect for two reasons.

First, it is not necessary for $k_{3}$ to be purely imaginary. In general it will be complex. In Ref. 1 it is stated that "... the component $k_{3}$ of the wave vector in the direction of the plane normal must be imaginary, since otherwise the impurity ['planar defect' is meant] would represent a source of outgoing waves, which is physically impossible." This argument does not dispose of complex $k_{3}$, as is well known even in the case of elastic continua. ${ }^{3}$ Second, it is not possible (except in unrealistically simple models) to satisfy the boundary conditions with a single $k_{3}$. Feuchtwang ${ }^{4}$ has shown that, in general, the displacements associated with a surface mode are actually given by

$$
\begin{aligned}
u_{\pi}\left(m_{3} ; \omega^{2}, \overrightarrow{\mathrm{k}}_{\rho}\right)= & \left.\sum_{g=-3 s H_{3}}^{-1} c_{g} \underset{\left(\omega^{2}\right.}{\left(\omega^{2}\right.}, \overrightarrow{\mathrm{k}}_{\rho}\right) \\
& \times u_{\pi}^{(g)}\left(e^{i k_{3}(g)} ; \omega^{2}, \overrightarrow{\mathrm{k}}_{\rho}\right) e^{i k_{3}^{(g)} m_{3}},
\end{aligned}
$$

outside the region perturbed by the surface [see Eq. (4.18) of Ref. 4], where the notation is defined in Ref. 4. In Eq. (2), $\mathrm{H}_{3}$ gives the range of the interaction between particles and $s$ is the number of particles per unit cell. It is thus necessary to include terms corresponding to $3 s H_{3}$ values of $k_{3}$ in the solution for a surface mode, rather than a single value of $k_{3} .^{5}$

The correct method for determining surface modes in a semi-infinite crystal has been given by Feuchtwang: Such modes must simultaneously satisfy the usual equation involving the bulk dynamical matrix

$$
\operatorname{det}\left[D_{\pi \sigma}(\overrightarrow{\mathrm{k}})-\delta_{\pi \sigma} \omega^{2}\right]=0,
$$

for each complex $k_{3}$ and the "resonance condition"

$$
\operatorname{det}\left[M_{\alpha \gamma}\left(\omega^{2}, \overrightarrow{\mathrm{k}}_{\rho}\right)\right]=0
$$

where the matrix $M_{\alpha \gamma}\left(\omega^{2}, \overrightarrow{\mathrm{k}}_{\rho}\right)$ is defined by Feuchtwang [see Eqs. (3.24), (4.6), (4.7), and (4.21) of Ref. 4]. Equation (4) is called a "resonance condition" for the following reason: Ordinarily an incoming propagating wave is scattered into a superposition of propagating and evanescent waves (not a single wave), according to Feuchtwang's Eq. (4. 1):

$$
\begin{aligned}
& u_{\pi}\left(m_{3} ; \omega^{2}, \overrightarrow{\mathrm{k}}_{\rho}, g\right)=N_{g}\left(\omega^{2}, \overrightarrow{\mathrm{k}}_{\rho}\right)\left(u_{\pi}^{(g)}\left(e^{i k_{3}^{(g)}} ; \omega^{2}, \overrightarrow{\mathrm{k}}_{\rho}\right)\right. \\
& \times e k_{3}^{\left(g^{g}\right)} m_{3}+\sum_{g^{\prime}=-3 s H 3}^{-1} R_{g g^{\prime}}\left(\omega^{2}, \overrightarrow{\mathrm{k}}_{p}\right) \\
& \times u_{\pi}^{\left(g^{\circ}\right)}\left(e^{i k_{3}^{\left(g^{\circ}\right)}} ; \omega^{2}, \overrightarrow{\mathrm{k}}_{\rho}\right) e^{i k_{3}^{\left(g^{\circ}\right)} m_{3}} \quad m_{3} \geq 2 H_{3} .
\end{aligned}
$$

If Eq. (4) is satisfied, however, then there is a solution with the form given in Eq. (2); i. e., the incoming wave is absent.

The assumption that surface modes involve only a single, purely imaginary $k_{3}$ is generally incorrect, ${ }^{6}$ and is incorrect in particular for crystals with long-range interactions such as $\mathrm{NaCl}$ crystals. The theory of Ref. 1 and the results of Ref. 2 are therefore invalidated by this assumption. This fact may account for some puzzling features of the results in Ref. 2, such as the disagreement with the results of Tong and Maradudin ${ }^{7}$ and the presence of 28 acoustic surface modes for one value of the two-dimensional wave vector $\overrightarrow{\mathrm{k}}_{\rho}{ }^{8}$

I wish to thank Dr. G. P. Alldredge for a number of helpful conversations and suggestions. 
*Work supported by the U.S. Air Force Office of Scientific Research under Grant No. AF-AFOSR 1257-67.

${ }^{1}$ B. N. N. Achar and G. R. Barsch, Phys. Rev. $\underline{188}$, 1356 (1969).

${ }^{2}$ B. N. N. Achar and G. R. Barsch, Phys. Rev. $\underline{188}$, 1361 (1969).

${ }^{3}$ J. L. Synge, J. Math. and Phys. 35,323 (1957).

${ }^{4}$ T. E. Feuchtwang, Phys. Rev. 155, 731 (1967).

${ }^{5}$ In anisotropic elastic continua, three values of $k_{3}$ are needed to satisfy the boundary conditions.

${ }^{6}$ Exceptions to this rule are certain unrealistically simple models, e.g., those used by Lengeler and Ludwig and by Takeno (see Ref. 1).

${ }^{7}$ S. Y. Tong and A. A. Maradudin, Phys. Rev. $\underline{181}$, 1318 (1969).

${ }^{8}$ See Table V of Ref. 2 for $\vec{k}_{\rho}$ along the [100] axis with magnitude $0.3 \times 2 \pi / r_{0}$.

\section{ERRATA}

High-Frequency de Haas-van Alphen Oscillations in Aluminum,

[Phys. Rev. B 2, 298 (1970)]. The $\kappa$ in the argument of the Bessel function, $J_{2}\left(2 \pi F \kappa h_{m} / H^{2}\right)$, on p. 305 has the value 0.43 instead of 0.23 .

Free-Carrier Radiation Peak in GaAs Due to Valence-Band Maxima Arising from Terms Linear in $k$, M. A. Gilleo and P. T. Bailey [Phys. Rev. 187,1181 (1969)]. The photoluminescence line at $1.520 \mathrm{eV}$ associated in this paper with valenceband maxima arising from terms linear in $k$ in addition to the other two lines in Fig. 1 have been found to be grating ghosts of stronger lines in the spectra. \{See the following erratum [Phys. Rev. B 3, $3581(1971)]$.

Free-Carrier and Exciton Recombination Radiation in GaAs, M. A. Gilleo, P. T. Bailey, and D. E. Hill [Phys. Rev. 174, 898 (1968)]. The following photoluminescence lines reported in this paper have been found to be grating ghosts of stronger lines in the spectrum: (i) the line at $1.5202 \mathrm{eV}$ associated in this paper with free-carrier recombination, (ii) the line at $1.5193 \mathrm{eV}$ associated with " $n=2$ " exciton recombination, and (iii) the pair of lines (Fig. 4) at 1.518 and $1.506 \mathrm{eV}$ related to the neutral zinc exciton line. In addition, the triplet shown in Fig. 10 became a doublet, and the doublet shown in Fig. 6 became a single line after this sample was etched. We believe the etch relieved some residual strain in the as-grown sample. Confirmation of this was obtained by examining other samples. The rest of the features of the spectra reported in this paper have been confirmed using different gratings.

Theory of Interband Raman Scattering in Semiconductors in Magnetic Fields, R. F. Wallis and D. L. Mills [Phys. Rev. B 2, 3312 (1970)]. The algebraic sign of the terms involving $m_{v}^{*}$ in Eq. (7) should be changed from plus to minus. In Eq. (8), the factor

$$
\left(\frac{\omega_{c}^{2}}{\omega_{1}^{2}-\omega_{c}^{2}}+\frac{\omega_{c}^{2}}{\omega_{0}^{2}-\omega_{c}^{2}}\right)
$$

should be replaced by the factor

$$
\left(\frac{\omega_{c} \omega_{1}}{\omega_{1}^{2}-\omega_{c}^{2}}-\frac{\omega_{c} \omega_{0}}{\omega_{0}^{2}-\omega_{c}^{2}}\right)^{2} .
$$

For $\mathrm{PbTe}$ at $50 \mathrm{kG}$ with YAG : Nd excitation, the scattering efficiency near resonance is little affected by this correction, but far from resonance it is increased by about two orders of magnitude over that shown in the right-hand side of Fig. 2. The possibility of experimental observation of the scattering far from resonance is thus significantly enhanced. We thank Dr. Y. Yafet for calling this correction to our attention. 